\title{
Effects of Organo-Modified Clay Addition and Temperature on the Water Vapor Barrier Properties of Polyhydroxy Butyrate Homo and Copolymer Nanocomposite Films for Packaging Applications
}

\author{
Okan Akin $^{1} \cdot$ Funda Tihminlioglu ${ }^{1}$
}

Published online: 20 April 2017

(C) Springer Science+Business Media New York 2017

\begin{abstract}
Polymer nanocomposites, based on bacterial biodegradable thermoplastic polyester, poly(hydroxybutyrate) (PHB), poly(hydroxyl-butyrate-co-hydroxyvalerate) (PHBHV), and commercial organo-modified montmorillonite (OMMT-Cloisite 10A) were prepared by solution casting method. This work aims to investigate the effect of Cloisite 10A type clay addition on the water vapour permeability properties of PHB/OMMT, and PHBHV/OMMT nanobiocomposite films. Temperature dependence of water vapor permeabilities of the films were also evaluated at various temperatures, and semi empirical permeability models were used to predict the permeability of polymer systems as a function of clay concentration and aspect ratio of nanoplates. Moreover, thermal, optical, and mechanical properties of the composites were examined by using varieties of techniques including differential scanning calorimeter (DSC), thermogravimetric analyzer (TGA), scanning electron microscope (SEM), and thin-film X-ray diffractometer (TF-XRD) respectively. Test results indicated that addition of highly intergallery swollen Cloisite 10A to the PHB/PHBHV, reduced the water vapor permeability up to 41 and $25 \%$ compared to native PHB and PHBHV films, respectively. Regarding the all mechanical properties measured, the maximum improvement was achieved for $3 \mathrm{wt} \%$ clay loaded samples for both PHB and PHBHV polymer composites. An increase of about 152 and $73 \%$ in tensile strength and of 77 and 18\%
\end{abstract}

Funda Tihminlioglu

fundatihminlioglu@iyte.edu.tr

Okan Akin

okanakin@iyte.edu.tr

1 Chemical Engineering Department, Izmir Institute of Technology, Urla, 35430 Izmir, Turkey in strain at break was achieved for PHB and PHBHV polymers, respectively. As a result of X-ray diffraction analysis, exfoliated structure was achieved at low clay loaded sample $(1 \% \mathrm{w} / \mathrm{w})$, however at higher concentration $(3 \% \mathrm{w} / \mathrm{w})$ the structure found as intercalated. Therefore, it is an evident that enhancement of characteristic properties highly depend on the dispersion level of clay particles in polymer matrix. The results obtained in this study show the feasibility of improvement of the properties of PHB based polymers with incorporation of nanoclay.

Keywords $\mathrm{PHB} \cdot \mathrm{PHBHV} \cdot$ Water vapor permeation kinetics $\cdot$ Cloisite 10A $\cdot$ Bionanocomposite $\cdot$ Mechanical properties

\section{Introduction}

Today, packaging is the largest sector in the plastics industry. As a result of growing population, there has been a significant increase in the demand for plastics being used in packaging applications. Synthetic petrochemical-based polymers have been widely used in a various packaging applications. Though great properties of these polymers exist, waste management becomes a problem due to their poor biodegradability [1]. Thus, biodegradable alternatives are urgently needed. There is growing interest in developing of biodegradable polymers as packaging materials to substitute of non-degradable polymer counterparts [2]. However, many research efforts showed that the properties are not still satisfactory for some applications and improvement of these properties are still challenging for industrial application point of view.

Polyhydroxyalkonates (PHAs) are aliphatic polyesters produced by microorganisms that are fully biodegradable 
and showed promising potential in packaging area. The most common types of PHA's are polyhydroxybutyrate (PHB) and polyhydroxybutyrate-co-hydroxyvalerate (PHBHV). Depending on the carbon source, type of produced PHA varies. They present good properties such as biodegradability, biocompatibility, and mechanical properties [3]. However, PHAs suffer from poor thermal stability, brittleness, barrier to gases and water vapor. Thus, attention has been focused on enhancement of PHA's properties by different kinds of ways. Most common and effective ways are preparation of PHA based blends or nano composites using inorganic or natural fillers to improve the mechanical and barrier properties of biodegradable polymers [4]. Zaikov and coworkers [5] studied the blends based on polyhydroxybutyrate with different hydrophilicity, namely polyethylene and polyamide. The blends were produced in an effort to adjust the resistance to hydrolysis through the control of water permeability. Miguel and his coworkers have worked on the carbon dioxide and water vapor transport properties of PHB blends with epichlorohydrin and epichlorohydrin copolymers [6].

Nano reinforcements of biodegradable polymers have strong promise in producing of environmentally friendly nanocomposites for packaging applications [4]. Layered silicate (clay) incorporated nanocomposites have received more attention due to the great enhancement in the properties of polymers [3, 7, 8]. Montmorillonite, hectorite and saponite are the most commonly used clay minerals for the preparation of polymer nanocomposites $[9,10]$. Numerous studies have shown that incorporation of even at small quantities of clay minerals enhanced the barrier, thermal and mechanical properties of biodegradable polymers $[3,11,12]$. Clay polymer interaction, which affects the dispersion level of clay in polymer matrix, is the key factor in nanocomposite structure. Depending on the dispersion level of layered silicates, the structure (exfoliated or intercalated structure) and properties are obtained [13]. Organic modification plays also important role in achieving good interaction between polymer and clay which affects the dispersion level [13]. Thus, attention has been focused on organically modified montmorillonite (OMMT) as nano reinforcement for proper selection of the functional group [14-16].

Although the preparation and structure-property relationship studies of PHA-layered silicate based nanocomposite films have been studied in the literature extensively $[7,17-20]$, only a few studies have been reported on the<smiles>C[N+](C)(C)Cc1ccccc1</smiles>

Fig. 1 Chemical structure of Cloisite 10A (HT is Hydrogenated Tallow ; 65\% C18; 30\% C16; 5\% C14. Anion: Chloride) [21] barrier properties of PHB-layered silicate nanocomposite films using modified montmorillonite (Cloisite 20A), kaolinite (Nanoter ${ }^{\mathrm{TM}} 2212$ and Nanoter ${ }^{\mathrm{TM}}$ 2000) [3], and Cloisite 30B for PHBHV polymers [12]. Therefore, in this study, effects of temperature and organomodified clay addition on the water vapour permeability properties of both PHB and PHBHV polymers were extensively evaluated. Experimental permeability data was also compared and fitted to several semi empirical permeability models. The research described in this article was also aimed to investigate the effect of addition of orgonamodified clay (Cloisite 10A), not studied in many polymer systems frequently in the literature, on thermal, mechanical, optical and surface properties of PHB and PHBHV nanocomposites films by connecting structure-property relationship.

\section{Experimental Section}

\section{Materials and Methods}

Poly (3-hydroxybutyrate) (PHB) and poly (hydroxybutyrate-co-valerate) (PHBHV) was purchased from Aldrich in powder form. Hydroxyvalerate concentration of PHBHV is $5 \mathrm{wt} \%$. Chloroform (Merck) was used as solvent. Organic modified montmorillonite, Cloisite 10A, was supplied by Southern Clay Products (Gonzales. TX). It is a natural modified montmorilonite with a quaternary ammonium salt (2MBHT: dimethyl. benzyl. hydrogenated tallow) (Fig. 1). The cation exchange capacity of Cloisite $10 \mathrm{~A}$ is $125 \mathrm{meq} / 100 \mathrm{~g}$ clay as given by the supplier.

The PHB or PHBHV-layered silicate composites were prepared by solution intercalation method. Firstly, polymer is dissolved in boiling chloroform by using reflux system. In order to increase the space between layers of slilicates, OMMT is swollen in chloroform for a day. After swelling process, PHB/OMMT and PHBHV/ OMTT nanocomposites were synthesized by sonicating the mixture for $1 \mathrm{~h}$. OMMT loading in polymer matrix (PHB and PHBHV) was changed as 1, 2, 3, and $5 \mathrm{wt} \%$. The composite films were then cast on glass petri dishes. To ensure complete removal of chloroform, films were kept in vacuum oven at $60^{\circ} \mathrm{C}$ and 0.5 bar vacuum for 3 days.

\section{Characterization}

X-ray diffraction (XRD) analysis was performed by using Phillipx X'Pert Pro MRD with $\mathrm{Cu} \mathrm{K} \alpha$ radiation $(\lambda=1.54 \mathrm{~nm})$ under a voltage of $40 \mathrm{kV}$ and a current of $40 \mathrm{~mA}$ over $2 \theta$ range of $0.25^{\circ}-10^{\circ}$. The dispersion of 
montmorillonite in polymer matrix was investigated by considering interlayer distance based on the disruption on crystal structure. The interlayer distance of OMMT was calculated by using Bragg's law.

Thermal stability of the polymer nanocomposite films was investigated by thermal gravimetric analysis (TGA). Tested films were heated from 30 to $1000^{\circ} \mathrm{C}$ at a heating rate of $10^{\circ} \mathrm{C} / \mathrm{min}$ with nitrogen flow of $30 \mathrm{~cm}^{3} / \mathrm{min}$.

Thermal properties of the samples were analysed by TA instrument Q10 differential scanning calorimetry (DSC). Samples were heated from 30 to $200^{\circ} \mathrm{C}$ with $10^{\circ} \mathrm{C} / \mathrm{min}$ heating rate and kept at $200^{\circ} \mathrm{C}$ for 5 min to eliminate the residual crystals of samples. In order to observe glass transition temperature, samples were cooled down to $-30^{\circ} \mathrm{C}$ with a cooling rate of $10^{\circ} \mathrm{C} / \mathrm{min}$. After cooling process, samples were heated to $400^{\circ} \mathrm{C}$.

Clay particle dispersion in polymer matrix was also observed by SEM (Philips XL-30-SFEG) analyses using a scanning transmission electron microscopy (STEM) detector. Diluted samples (1:10,000 v/v chloroform solution) of 1 and $3 \mathrm{wt} \%$ PHB/OMMT were dropped on grid.

The mechanical properties of prepared films were measured using a texture analyzer (TA XT Plus) equipped with a $5 \mathrm{Kgf}$ load cell in tensile mode according to ASTM D-882 standard. Samples were prepared at $10 \mathrm{~mm}$ in width and $100 \mathrm{~mm}$ in length and preconditioned at $23^{\circ} \mathrm{C}$ and $\% 50 \mathrm{RH}$ for $24 \mathrm{~h}$ before testing. Initial gauge length and cross-head speed were set as 100 and $10 \mathrm{~mm} / \mathrm{min}$, respectively. Tensile strength, Young's Modulus, and elongation at break of the films were calculated from strain-stress data.

The color change due to addition of clay particles into PHB and PHBHV polymers were investigated using a colorimeter (Avantes). Native polymer films were used as reference background. Based on Hunter method; L, a, and $\mathrm{b}$ values were averaged from three readings for each film. Readings from horizontal and vertical axis represented as $\mathrm{L}$, $\mathrm{a}$, and $\mathrm{b}$ values respectively. $\mathrm{L}$ indicates the lightness ranging from black to white. The $\mathrm{a}$ and $\mathrm{b}$ values are the chromatic coordinates (ranging from a: greenness to redness and b: blueness to yellowness). The total color difference $(\Delta \mathrm{E})$ can be calculated with the following Eq. (1).

$\Delta E=\sqrt{\Delta L^{2}+\Delta a^{2}+\Delta b^{2}}$

Contact angle measurements of PHB-PHBHV nanocomposite films were carried out by means of Attention Theta Optical Tensiometer, KSV. The surface wettability property of films was performed by dropping $6 \mu$ of water on the film surface. Images of water droplet were recorded in trigger mode. Each measurement was evaluated by taking ten images of water droplet at one second interval. At least ten measurements at random places of surface of the films were reported. Left and right side of contact angle of water droplet on nanocomposite films were analyzed via the software of device.

\section{Barrier Properties: Water Vapor Permeation Measurements}

The effects of addition of Cloisite 10A and temperature on the barrier performance of PHB homo and copolymer films were investigated by water vapor permeability analyzer (Mocon Permatran 3/33). Water vapor permeability (WVP) of nanocomposites was determined according to ASTM F1249 standard at $37.8^{\circ} \mathrm{C}$ and $90 \%$ RH. Test film was placed between two test cells. Nitrogen as a carrier gas was passed through ultra pure water to adjust the RH and flowed to the test film with $100 \mathrm{~cm}^{3}$ per minute. Data were recorded as water vapor transmission rate (WVTR) $\left(\mathrm{g} / \mathrm{m}^{2}\right.$ day). All the results related to water vapor permeability shown in the present work represent the average of at least two or three independent experimental measurements. Permeability of the samples ( $\mathrm{g}$ water vapour* thickness $(\mathrm{mm})$ per area $\left(\mathrm{m}^{2}\right) *$ time (day) *pressure $(\mathrm{mmHg}))$ was calculated using Eq. 2.

Permeability $=\frac{W V T R}{S\left(R_{1}-R_{2}\right)} * L_{\text {fim }}$

$\mathrm{R}_{1}$ is relative humidity at the source expressed as a fraction $\left(\mathrm{R}_{1}=0.9\right.$ for $90 \% \mathrm{RH}$ chamber $)$. $\mathrm{R}_{2}$ is relative humidity of the vapor sink expressed as a fraction $\left[R_{2}=0\right.$ for the $0 \% \mathrm{RH}$ chamber (dry side)]. $\mathrm{S}$ is the vapor pressure of water at the test temperature in $\mathrm{mm}$ of mercury and $\mathrm{L}_{\text {film }}$ is the thickness of the tested film. For each film sample, three measurement were done and the average was reported as the water vapor permeability data. Moreover, temperature dependence of water vapor permeabilities of PHB and PHBHV nanocomposite films were evaluated at $10,20,30$ and $40^{\circ} \mathrm{C}$.

Thickness of tested films was measured with an electronic digital micrometer (293-821. Mitutoyo) with $1 \mu \mathrm{m}$ sensitivity. Thicknesses were determined by taking the average of ten different measurements that randomly reckoning from test films.

\section{Results and Discussion}

\section{Morphological Characterization}

XRD patterns of Cloisite 10A and the prepared nanocomposite films are shown in Figs. 2 and 3. The basal spacing (d-spacing) of Cloisite 10A has been calculated as $1.92 \mathrm{~nm}$ with a diffraction peak at $2 \theta=4.7^{\circ}$ by using Bragg equation (Eq. 3). The position obtained for Cloisite 10A peak was in 


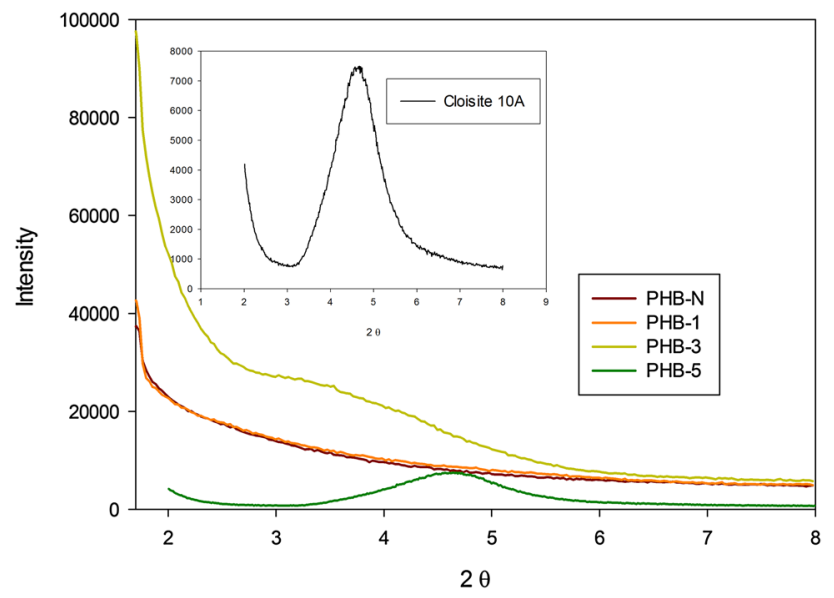

Fig. 2 XRD patterns of Cloisite 10A, native PHB, and exfoliated, intercalated structure of layered silicates of PHB-1, PHB-3 (1-3 wt \% OMMT) and PHB-5 (5wt\% OMMT) nanocomposite films, respectively

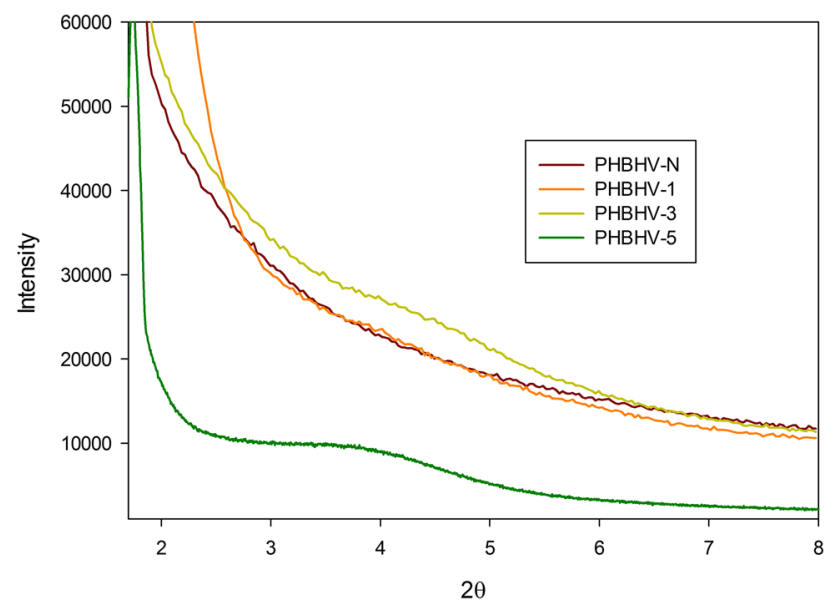

Fig. 3 XRD patterns of native PHBHV and exfoliated structure of PHBHV-1, PHBHV-3 (1-3 wt\% OMMT) and intercalated structure of PHBHV-5 (5 wt\%OMMT) nanocomposite films

agreement with the value $(19.2 \AA)$ reported by the supplier company.

$\lambda=2 d \sin \theta$

Since XRD result of neat polymer samples (PHBHV-N and PHB-N) indicated no characteristic peak in the scanning range of $1^{\circ}-9^{\circ}$ (Figs. 2, 3), structure of clay layers in the polymer matrix can be assessed by XRD analysis. Characteristic peak of Cloisite 10A was disappeared for $1 \mathrm{wt} \%$ clay loaded samples (PHBHV-1, PHB-1). The vanishing $\mathrm{X}$-ray diffraction pattern of Cloisite $10 \mathrm{~A}$ is due to exfoliation of silicate layers into polymer matrix. Clay stacks open up and clay platelets disperse disorderly, therefore it is decreasing the XRD reflection and disappeared in exfoliated structures. Many studies showed that exfoliation of silicate layers are achieved at lower clay content loadings $[22,23]$. Broadened characteristic peak was observed for the X-ray diffraction patterns of 3 and $5 \mathrm{wt} \%$ loaded samples of PHB and PHBHV polymers. Shifting of characteristic peak is attributed to increase in d-spacing of layer silicates. As the d-spacing increases, the dispersion level of layered silicate increases as well. However for broadened peaks, structure turns in to more intercalated structure with lower stack openings. The characteristic peak of Cloisite 10A $\left(2 \theta=4.5^{\circ}\right)$ broadened at $3 \mathrm{wt} \%$ of PHB and PHBHV nanocomposites. Therefore, it is not possible to calculate the interlayer distance for $3 \mathrm{wt} \%$ OMMT loaded samples. Therefore, the samples of PHB-3 and PHBHV-3 can be interpreted as in partially/fully exfoliated and intercalated structure. Due to the poor dispersion of silicate layers at higher OMMT loading (5 wt\%), characteristic peak of Cloisite 10A became obvious. Bragg's law calculations show that interlayer distance of PHB/ OMMT (5 wt \%) and PHBHV (5 wt\%) was found as 1.92 and $2.32 \mathrm{~nm}$ respectively.

The level of dispersion was also investigated by STEM. Figure 4 illustrates the STEM images of $1-3 \mathrm{wt} \%$ PHB/ OMMT samples at $100000 \mathrm{X}$ magnifications. The clay layers correspond to dark lines and polymer corresponds to grey area. Good dispersion of clay particles in polymer matrix is clearly seen from images. Clay particle size varies from 100 to $900 \mathrm{~nm}$ in length and from 50 to $300 \mathrm{~nm}$ in width for both 1 and $3 \mathrm{wt} \%$ OMMT loaded PHB nanocomposites.

XRD analysis show that clay loading above $3 \mathrm{wt} \%$ samples (PHB/OMMT and PHBHV-OMMT), d-space is not changed significantly. Thus, STEM images also confirmed by XRD analysis in a way that clay particles seem in stacks of silicate layers which define its dispersion level. Mostly exfoliated structure was observed, this result is in good agreement with XRD and explains the lack of clay spectra in the main spectra.

\section{Water Vapor Permeability Calculations}

Effect of clay loading on water vapor barrier property of PHB and PHBHV films were studied. As seen in the Fig. 5, the continuous decrease in water vapor permeability of PHB/OMMT and PHBHV/OMMT nanocomposite films was observed with an increase in OMMT loading up to 2 and $3 \mathrm{wt} \%$, respectively. Generally, a critical limit of clay content was observed due to the changes in nanostructure after certain nanoclay loadings. This behaviour was also reported by many researchers in the literature for many polymer-layered silicate systems [7, 16]. Moreover, the lowest permeability value was achieved at 2 and $1 \mathrm{wt} \%$ clay 

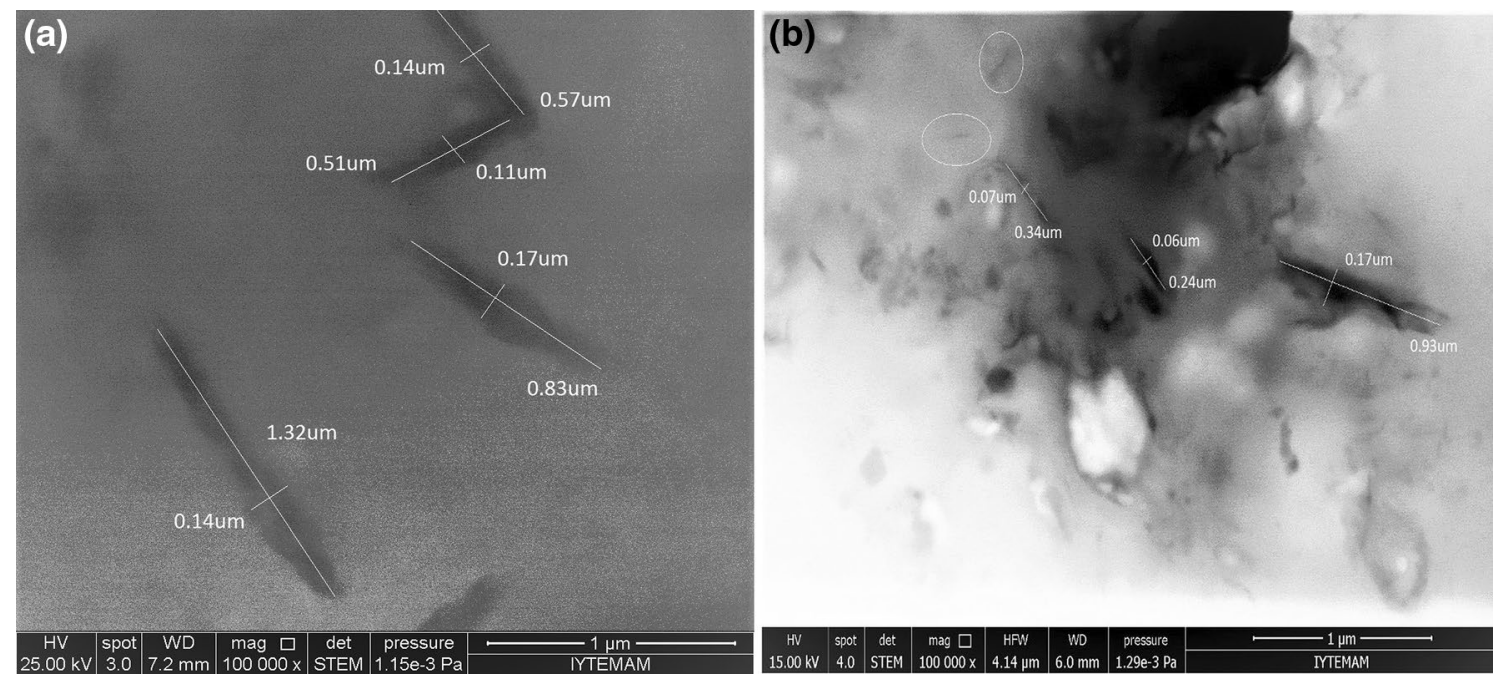

Fig. 4 STEM images of 1, 3 wt\% of PHB/OMMT nanocomposite films and measured dimensions (length/width) of distributed layers within the matrix

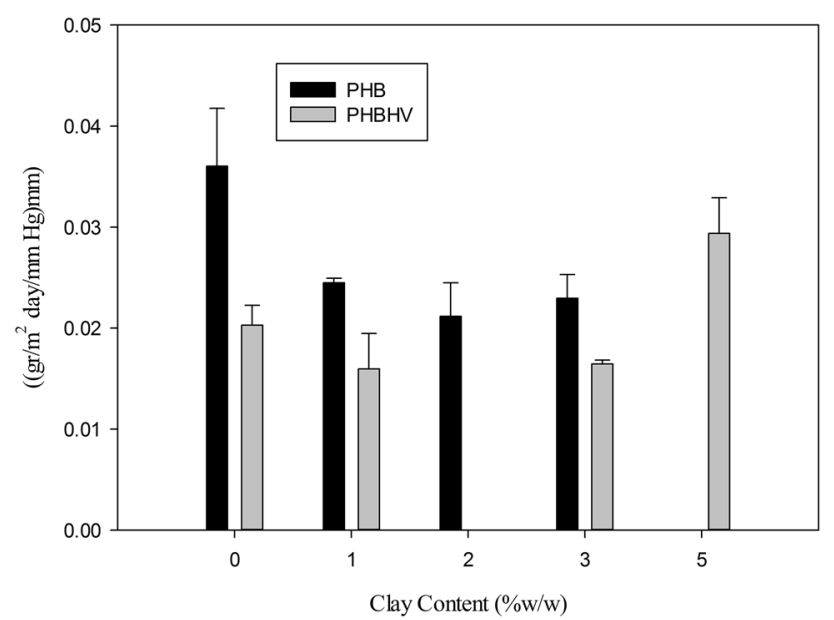

Fig. 5 Water vapor permeability of PHB/OMMT, PHBHV/OMMT nanocomposite films at different clay contents

loaded for PHB and PHBHV nanocomposites, respectively. Depending on the amount of clay loaded and its dispersion level, WVP of pure PHB and PHBHV decreased by 41 and 25\%, respectively. The study of Sanchez et al. [3] on PHB blend/OMMT system showed that the barrier improvement of water vapor molecules in PHB blend-clay composites (4 wt $\%$ ) was $46 \%$ and resulted from reductions in diffusion due to the tortuosity effect, and also solubility coefficients of water vapor in PHB blend composites [3]. Our study gave similar reduction of about $41 \%$ for only $2 \mathrm{wt} \%$ OMMT loading. However, Cretois et al. [12] showed an increase in water vapor permeability due to the increase in gas solubility. Improvement of water vapour performances of the PHB and PHBHV polymers could be interpreted as a result of layered silicate dispersion either in fully or partially exfoliated in polymer matrix. Exfoliated structure of layered silicates resulted in the formation of high aspect ratio (length/ width) fillers within the matrix in which more tortuous path is created. An increase in the tortuosity is the main factor that effect the water vapor barrier performance of nanocomposites. Beside the tortuosity effect, addition of filler in polymer matrix resulted in decrease of free volume of polymer that effects the solubility of water vapor and hence barrier performance of nanocomposites. Layered silicates also effect the crystallinity and crystal size of spherulites in case of PHB/PHBHV nanocomposites which may be attributed to the enhancement in barrier performance of nanocomposites. As water vapor permeability and XRD data are in good agreement each other and confirm the structure of fully and partially exfoliated structure of PHB (2 wt \%) and PHBHV (3 wt\%), decrease in permeability could be mainly attributed to tortuous path formed by dispersed clay layers within the polymer matrix. These impermeable clay layers, therefore, increase the diffusion length of water vapour molecules through the polymer matrix and causing decrease of diffusion coefficient $[24,25]$. Therefore, it is an evident that degree of exfoliation is an important parameter for silicate layered nanocomposites systems [13]. To understand the level of exfoliation for layered silicates, at this point, aspect ratio must also be considered for the interpretation of permeability since the agglomerations effect on the aspect ratio and as well as the diffusion pathway of water vapor. Therefore, semi empirical permeability models can be used to predict the permeability of polymer systems as a function of clay concentration and aspect ratio of nanoplates. Based on different assumptions such as filler geometry and orientation in polymer matrix, several 
models were developed in literature to describe the permeability of diffusing molecule in filled polymers (Table 1). Significant improvement in barrier properties was achieved via dispersion of layered silicates in polymer matrix which formed a tortuous path due to high aspect ratio of filler. In order to evaluate aspect ratio, models were fitted to experimental data with a general assumption that nanoparticles are impermeable to penetrant molecule and permeating molecules are forced to wiggle around filler and hence diffuse through a tortuous pathway. Increment in aspect ratio resulted in the decrease in area available for diffusion, a result of impermeable filler replacing with permeable polymer hence permeability decreases [26].

In the permeability equations, two important parameters; $\alpha$ and $\varnothing$ are the aspect ratio and volume fraction of clay platelets in the nanocomposites, respectively. $\mathrm{P}$ and $\mathrm{P}_{0}$ denotes for the permeabilities of pure polymer and polymer nanocomposite. Permeability models employed to predict the aspect ratio for PHB and PHBHV nanocomposites by fitting the experimental data are shown in Fig. 6 for water vapor permeation through PHB and PHB/OMMT nanocomposites. The volume fractions of clay in nanocomposites $(\varnothing)$ were calculated by using the Eq. (4),

$$
\theta=\frac{1}{1+\left(\rho_{c} \frac{(1-M c}{\rho_{p} M c}\right)}
$$

where $\rho_{c}, \rho_{p}$ and $M_{c}$ denote for density of nanoclays, density of polymer, and mass percentage of nanoclays in the polymer matrix, respectively. As seen in Fig. 6, the Cussler Regular and Cussler Random models gave the best fitting curve to experimental WVP data of PHB nanocomposite films. The models, Cussler Regular and Random models, gave aspect ratios around 160 and 240, respectively for

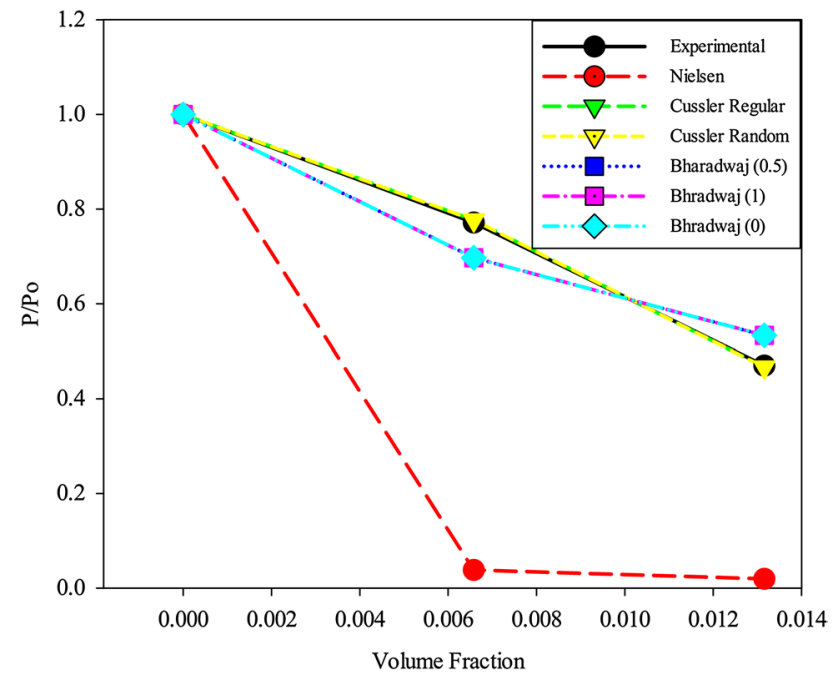

Fig. 6 Calculated relative WVPs based on permeability models of Nielsen, Cussler, and Bhradwaj and experimental relative WVPs of PHB/OMMT nanocomposite films

Table 1 Permeability models applied to WVP data [27]

\begin{tabular}{|c|c|c|c|c|}
\hline Models & Filler type \& orientation & Formulas & Assumptions & Reference \\
\hline Nielsen & regular array, oriented & $\frac{P}{P_{o}}=\frac{1-\phi}{1+(\underline{\underline{\alpha}}) \phi}$ & 2D, rectangular platelets, regular array, platelets align & Nielsen [28] \\
\hline Cussler-regular array & regular array, oriented & $\frac{P}{P}=\frac{1-\phi}{\alpha \phi^{2}}$ & $2 \mathrm{D}$, rectangular platelets, regular array, platelets align & Eitzman et al. [29] \\
\hline Cussler random array & random array, oriented & $\frac{P}{P_{o}}=\frac{1-\phi}{\left(1+\frac{\alpha \phi}{3}\right)^{3}}$ & $\begin{array}{l}2 \mathrm{D} \text {, rectangular platelets, random array, platelets align } \\
\text { normal to diffusion }\end{array}$ & Lape et al. [30] \\
\hline Bharadwaj & $\begin{array}{l}\text { random array, non- } \\
\text { oriented }\end{array}$ & $\frac{P}{P_{o}}=\frac{1-\phi}{1+\frac{\alpha \phi}{3}\left(S+\frac{1}{2}\right)}$ & $\begin{array}{l}\text { 2D, rectangular platelets, random array, platelets align } \\
\text { different directions to diffusion direction }\end{array}$ & Bharadwaj [31] \\
\hline
\end{tabular}

$\alpha=\mathrm{L} / 2 \mathrm{~W}$

L: For square fillers of length/width of the filler

W: Thickness of the filler

$\phi$ : The volume fraction of the filler 


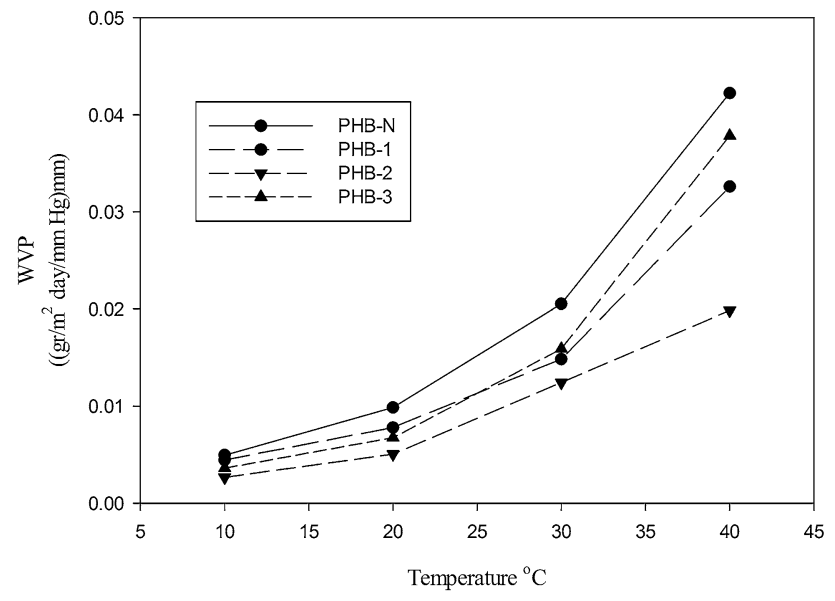

Fig. 7 Temperature dependence of WVP of PHB nanocomposite films within the temperature interval of $10-40^{\circ} \mathrm{C}$

Table 2 Slope and $\mathrm{R}^{2}$ values of Arrhenius plot and WVP activation energies of PHB nanocomposites films

\begin{tabular}{llll}
\hline Sample & R-squared & Slope & Ea $(\mathrm{kJ} / \mathrm{mol})$ \\
\hline PHB-N & 0.9983 & -6350.1 & 52.79 \\
PHB-1 & 0.9888 & -5849.9 & 48.64 \\
PHB-2 & 0.9894 & -6147.1 & 51.11 \\
PHB-3 & 0.9895 & -6992.5 & 58.14 \\
\hline
\end{tabular}

WVP data, which is in good agreement with the reported aspect ratio range (10-1000) for layered silicates [16, 32]. Moreover, Sanchez-Garcia and coworkers evaluated aspect ratio of layered silicates in PHB matrix via TEM analysis, and they found that average aspect ratio was greater than 100 which is consistent with the results found by Cussler Regular and Random models [3].

Moreover, temperature dependence of water vapor permeabilities of PHB and PHBHV nanocomposite films were evaluated at $10,20,30$ and $40^{\circ} \mathrm{C}$. The temperature dependence on gas transport through a polymer generally follows Arrhenius relation (Eq. 5) that shows a linear relationship between a transport parameter logarithm and the reciprocal of temperature (T). By plotting Arrhenius plots, activation energies of nanocomposites were evaluated.

$P=P_{0} e^{-E_{a} / R T}$

WVPs of PHB nanocomposites at different temperatures were shown in Fig. 7. An exponential increase in permeability with increasing temperature was observed. This behaviour can be resulted from dependence of WVP to both temperature and partial pressure of water vapor across the sample. Therefore, pressure difference across the sample at higher temperature increases hence, as seen in Fig. 7, it is an evident that the deviations among WVPs of PHB nanocomposites were higher at test temperature of $40^{\circ} \mathrm{C}$ when compared to lower test temperatures. These findings are also in good agreement with Hulsmann and coworkers study [33]. The lowest permeability was found at $10^{\circ} \mathrm{C}$ as expected due to the lowest partial pressure of water vapor among the test temperatures tested.

The activation energy (Table 2) describes the acceleration of permeation by temperature. Cloisite $10 \mathrm{~A}$ addition at low content ( 1 and $2 \% \mathrm{w} / \mathrm{w})$ resulted in decrease at activation energy. In other words, pristine PHB-N is more temperature dependent in permeation of water vapor then clay containing PHB-1 and PHB-2 samples. This behaviour can be explained by barrier performance of layered silicates. The $2 \mathrm{wt} \%$ PHB nanocomposites exhibited the best results in terms of temperature dependency for, fully exfoliated structure of layered silicates increased the tortuosity and free volume of polymer matrix. In addition, relaxation of polymer chains is restricted by layered silicates which are well dispersed in polymer matrix. Therefore, free volume of polymer created by holes was prevented via layered silicates. In addition, this free volumes can cause cluster affect which increases permeability of water vapor in polymers [26]. The sample PHB-3 showed the highest activation energy with a value of $58 \mathrm{~kJ} / \mathrm{mol}$. It is an evident that dispersion of layered silicates affect activation energy of water vapor permeability. It was proofed that exfoliated structure was formed at low content $(1 \% \mathrm{w} / \mathrm{w})$ of Cloisite $10 \mathrm{~A}$ and exfoliation resulted in better interaction with polymer chains. This behaviour is also in good agreement with findings of activation energy values.

The temperature dependent water vapor permeability values of PHBHV nanocomposites were also evaluated via permeation tests at different temperatures (Fig. 8). As shown in PHB measurements, PHBHV samples exhibited similar trend in activation energy (Table 3); exfoliated structure was obtained at $1 \% \mathrm{w} / \mathrm{w}$ Cloisite $10 \mathrm{~A}$ loading that is resulted in decrease in activation energy. In other words, water vapor permeation in PHBHV-N sample was more temperature dependent then PHBHV-1. This could be resulted due to restriction of mobility of polymer chains via strong interaction between layered silicates and polymer chains [33]. However, PHBHV-5 exhibited higher activation energy then pristine PHBHV. This could be resulted due to intercalated structure, which allows chain mobility, of Cloisite $10 \mathrm{~A}$ in polymer matrix.

The permeation process is the mass transport dependent on time through a solid piece of material with at least two surfaces acting as interfaces to the surrounding gas. Therefore, when the activation energy of water vapor permeation is considered, the mechanism of permeation should be taken into account. There are four steps occurred while a gas molecule penetrates through a membrane; (i) 


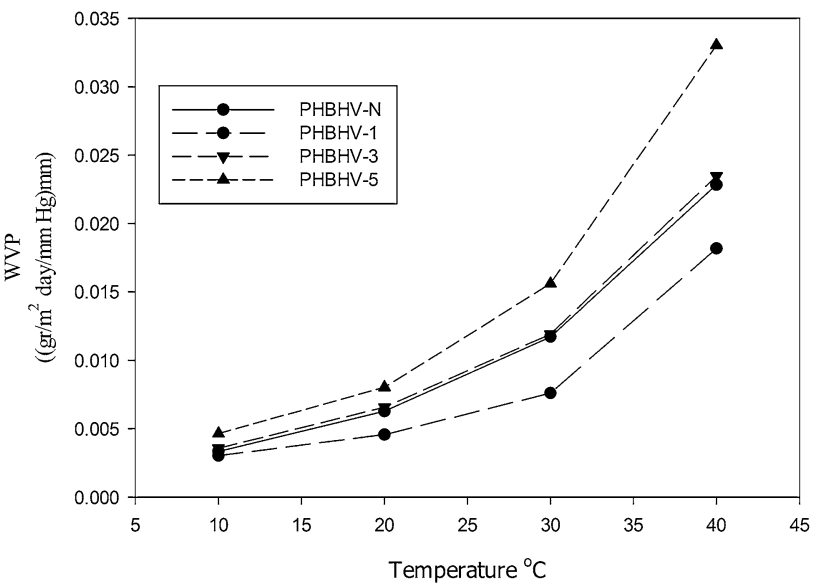

Fig. 8 Temperature dependence of WVPs of PHBHV nanocomposite films within the temperature of $10-40{ }^{\circ} \mathrm{C}$

Table 3 Slope and $\mathrm{R}^{2}$ values of Arrhenius plot and WVP Activation energies of PHBHV nanocomposites films

\begin{tabular}{llll}
\hline Sample & R-squared & Slope & Ea $(\mathrm{kJ} / \mathrm{mol})$ \\
\hline PHBHV-N & 0.9981 & -5655.1 & 47.01 \\
PHBHV-1 & 0.9575 & -5190.8 & 43.16 \\
PHBHV-3 & 0.9900 & -5790.4 & 48.14 \\
PHBHV-5 & 0.9968 & -5925.9 & 49.94 \\
\hline
\end{tabular}

The adsorption on top of one surface (ii) absorption inside the material (iii) diffusion (iv) desorption out of the material. Layered silicate effect on these four steps in water vapor permeation that should be considered. For instance, the adsorption on top of one surface can be related to surface characteristics such as hydrophobic nature which was evaluated via contact angle measurements. The absorption of water vapor inside the material is related to dissolution obeying Henry's law plus "hole filling" obeying Langmuir expression. However, the gas molecules are only soluble in amorphous phase which can be related to see the effect of layered silicate on crystallization kinetic of nanocomposites films [26].

\section{Thermal Properties}

DSC and TGA analysis were applied to PHB and PHBHV nanocomposite films for determination of effect of clay loading on thermal stability as well as melting and crystallization behaviour. DSC thermograms of pure PHB and the nanocomposites, from 1 to $5 \mathrm{wt} \%$ clay loaded PHB/ OMMT nanocomposites, are presented in Fig. 9. There are two endothermic peaks between 150 and $180^{\circ} \mathrm{C}$ due to the possible mechanisms: different morphology (crystal structures, and lamellar thickness), crystal

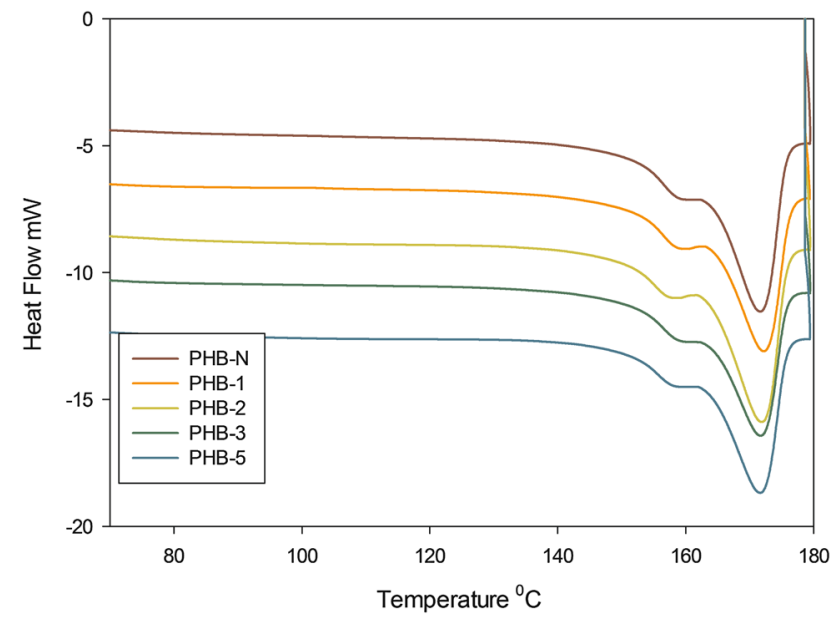

Fig. 9 DSC thermograms: first heating runs for PHB-N and PHB/ OMMT (1-7\%) nanocomposites

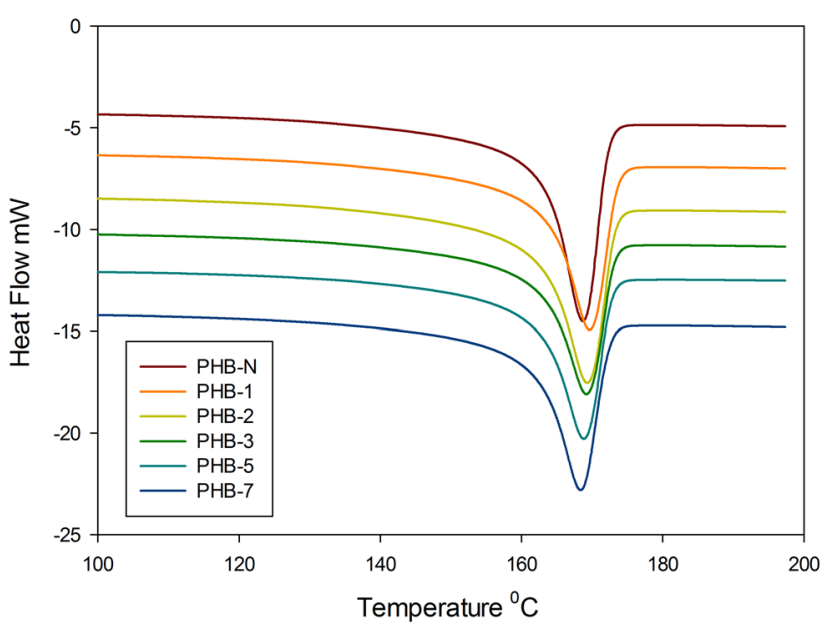

Fig. 10 DSC thermograms: second heating runs for PHB-N and PHB/OMMT (1-7\%) nanocomposites

modifications, melting, recrystallization and remelting during heating. As seen in Fig. 9, the first minor peak follows the second major peak. When DSC experiments were performed at two times, as observed in Fig. 10, second run of DSC thermograms of the films showed that minor peak disappeared and melting temperature at second heating was found to be lower is lower than first heating melting, $\mathrm{Tm}_{2}<\mathrm{Tm}_{1}$ for instance, PHB-1 with first and second melting temperatures of 172.3 and $169.63^{\circ} \mathrm{C}$, respectively (Table 4; Fig. 10). The same effect was also observed by Botana and coworkers [6]. This could be resulted from first heating run which caused the elimination of crystal history of samples. Thus, there is only one endothermic peak at second heating run (Fig. 9). 
These findings are in accordance with the literature findings that resulted from melting of crystals with different lamellar thickness [9]. Thus, clay addition to the polymer matrix prevented the creation of thicker lamellar crystals. This may be related to a slight reduction in crystal size and restricted polymer chain mobility in the presence of filler [9, 34]. The addition of Cloisite 10A generally did not change much the total crystallinity percentage of PHB and PHBHV nanocomposites, except at $1 \mathrm{wt} \%$ OMMT loaded nanocomposites (Table 4).

As the clay loading increased, no significant change in melting temperature of PHB nanocomposites (Table 4) was observed that is consistent with the findings of the study of Sancez-Garcia and coworkers [3]. However, the second melting temperature of native PHBHV-N increased from 153.05 to $159.4{ }^{\circ} \mathrm{C}$ with the addition of $1 \mathrm{wt} \%$ (PHBHV1) of clay addition. This could be due to restriction of mobility of the polymer chains that resulted in the higher melting temperature for second heating run. The differences between PHB and it copolymer PHBHV may be due to the interfacial interaction of copolymer chain with the clay particles. In addition, enthalpies of high concentration of clay loaded samples were slightly different. This could be resulted from agglomerates caused non uniformity of samples. Melting enthalpies at second heating run showed that clay acted as a nucleating agent at low concentration (1 wt $\%$ ) supported by the trend of crystallinity change. Contrary, at higher concentration of clay loaded samples, agglomerates restricted the polymer chain mobility resulted in lower crystallinity and melting enthalpy [14].

The cold crystallization temperature $\left(\mathrm{T}_{\mathrm{cc}}\right)$ of samples was also evaluated during the cooling step of melted samples (Table 4). As it is seen, generally the addition of clay to polymer resulted in a very slight decrease $\left(2-4^{\circ} \mathrm{C}\right)$ in the cold crystallization temperature of pristine polymer except for high clay loaded samples of PHBHV (PHBHV3 , PHBHV-5). Although addition of nanoclay in some polymer systems generally resulted a nucleation effect. Our results do not support this finding. The value of $T_{c}$ for PHBHV-N reduced with the addition of $\mathrm{HV}$ unit in the polymer structure (PHB) from 114.12 to $102.96^{\circ} \mathrm{C}$. It could be resulted due to presence of $\mathrm{HV}$ content offering a larger free volume of molecular movement. As regarding the thermal properties of PHB based nanocomposites, it can be said that the change of the water vapour properties is not effected by the change of the crystallinity. It is mainly dominated by the dispersion of nanoclay structure in the polymer matrix.

\section{Mechanical Properties}

The effect of clay addition on the mechanical properties of the PHB and PHBHV nanocomposite films were investigated. The results of mechanical tests are presented in Fig. 11 as tensile strength (TS) (11-a), \% elongation at break (EB) (11-b) and Young's Modulus (YM) (11c) of pure polymer and its nanocomposites. As seen in Fig. 11a, the native PHB had a tensile strength of $11.6 \mathrm{MPa}$ and strain at break of $0.43 \%$. Even a $1 \mathrm{wt} \%$ clay addition increased tensile strength of PHB nanocomposites at about $156.1 \%$. Moreover, Young's modulus, in other word stiffness, and \% elongation at break of pristine PHB was also enhanced at about 54 and $90 \%$, respectively. The mechanical properties of both polymers increased with the addition of clay loading. Regarding the all properties measured, the maximum improvement was achieved for $3 \mathrm{wt} \%$ clay loaded samples for both PHB and PHBHV polymer composites. An increase of about 152.3 and $73.2 \%$ in
Table 4 DSC data of neat PHB, PHBHV and PHB/ OMMT, PHBHV/OMMT nanocomposite films

\begin{tabular}{llllllll}
\hline Sample name & $\mathrm{T}_{\mathrm{m} 1}\left({ }^{\circ} \mathrm{C}\right)$ & $\Delta \mathrm{H}_{\mathrm{m} 1}(\mathrm{~J} / \mathrm{g})$ & $\mathrm{T}_{\mathrm{m} 2}\left({ }^{\circ} \mathrm{C}\right)$ & $\Delta \mathrm{H}_{\mathrm{m} 2}(\mathrm{~J} / \mathrm{g})$ & $\mathrm{T}_{\mathrm{cc}}\left({ }^{\circ} \mathrm{C}\right)$ & $\Delta \mathrm{H}_{\mathrm{c}}(\mathrm{J} / \mathrm{g})$ & Crystallinity $\%$ \\
\hline PHB-N & 172.1 & 91.9 & 168.7 & 90.1 & 114.1 & 83.7 & 61.7 \\
PHB-1 & 172.3 & 94.9 & 169.6 & 95.9 & 114.0 & 86.6 & 65.7 \\
PHB-2 & 172.9 & 89.1 & 169.3 & 91.5 & 111.4 & 79.6 & 62.6 \\
PHB-3 & 172.2 & 87.1 & 169.2 & 89.5 & 112.9 & 80.5 & 61.3 \\
PHB-5 & 171.7 & 83.8 & 168.8 & 85.8 & 111.9 & 76.1 & 58.7 \\
PHBHV-N & 153.4 & 16.4 & 153.0 & 33.1 & 102.9 & 68.3 & 49.4 \\
PHBHV-1 & 152.0 & 17.7 & 159.4 & 24.2 & 97.7 & 62.1 & 54.9 \\
PHBHV-3 & 152.05 & 10.8 & 160.6 & 32.0 & 104.7 & 66.1 & 48.4 \\
PHBHV-5 & 152.1 & 12.3 & 160.3 & 26.3 & 105.2 & 65.4 & 45.6 \\
\hline
\end{tabular}

$\mathrm{Tm}_{1}$ : Melting temperature determined at first run

$\mathrm{Tm}_{2}$ : Melting temperature determined at second run

Tc: Cold Crystallization temperature

$\Delta \mathrm{H}_{\mathrm{m} 1}$ : Melting enthalpy of first run

$\Delta \mathrm{H}_{\mathrm{m} 2}$ : Melting enthalpy of second run

$\Delta \mathrm{H}_{\mathrm{c}}$ : Crystallization enthalpy 

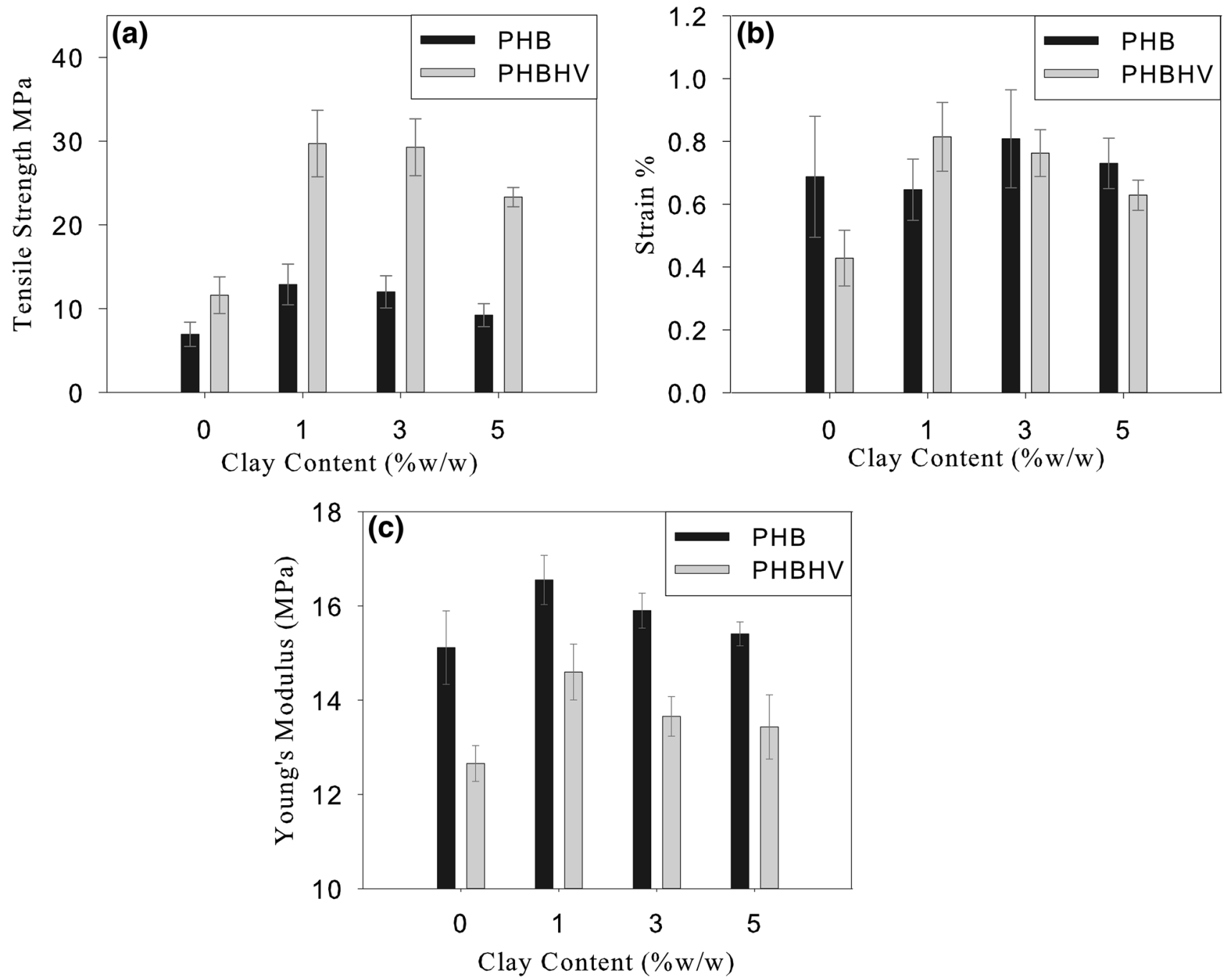

Fig. 11 a Tensile strength, b strain, and $\mathbf{c}$ Young Modulus of PHB/OMMT and PHBHV/OMMT nanocomposite films

tensile strength and of strain at break 77.4 and $17.5 \%$ was achieved for PHB and PHBHV polymers, respectively. The increment was much higher for PHB polymer than that of PHBHV. To our knowledge, the improvement obtained in the mechanical properties obtained in this study was the highest among the other commercial clays studied in the PHB and PHBHV polymers PHBHV-Cloisite 30B -8.2\% TS, -2\% EB, \%45 YM [19]; PHBHV-Cloisite 30B 3.2\% TS, (-10.5) \% EB, \%15.3 YM [20]; PHB-Cloisite 30B $16.3 \%$ TS, 21\% EB, 5\% YM; PHBHV -30B 6\% TS, \%85 EB, (-4) \% YM [17]; PHB-Cloisite 15A (-13.1\%)TS, $2.9 \% \mathrm{~EB},(-80.3) \%$ YM [18].

Dispersion plays key role in the improvement of mechanical properties of polymer matrix composites. The enhancement in mechanical properties of the nanocomposite films can be attributed to achievement of intercalated/exfoliated structures [13]. In fact, a good dispersion of nanofillers in the polymer tends to improve the tensile strength and strain at break. The stiffness of polymer nanocomposites generally increases with the nanoparticles volume fraction, as long as a sufficient dispersion and degree of exfoliation of these particles are ensured. Therefore, the increase in the mechanical properties of PHB and PHBHV composites was greater at a low Cloisite 10A content; this indicates that the fillers were better dispersed and exfoliated. However, our results showed that after $3 \mathrm{wt} \%$ of clay loading, mechanical properties decreased for both PHB and PHBHV composites which could be resulted from the agglomeration of clay particles in polymer matrix. Despite of agglomeration affect, high loaded samples (5 wt $\%$ of clay loaded samples) showed higher mechanical properties than pure PHB and PHBHV films. The effect of clay addition on mechanical properties is in good agreement with water vapor permeability and XRD results. 
Table 5 Color change measurements of PHB/OMMT, PHBHV/ OMMT nanocomposite films

\begin{tabular}{lllll}
\hline Sample Code & $\mathrm{L}$ & $\mathrm{a}$ & $\mathrm{b}$ & $\Delta \mathrm{E}$ \\
\hline PHB-N & 87.38 & -0.72 & 2.92 & 0.00 \\
PHB-1 & 86.25 & -0.59 & 3.54 & 1.29 \\
PHB-2 & 86.94 & -0.63 & 3.44 & 0.70 \\
PHB-3 & 86.61 & -0.58 & 3.41 & 0.94 \\
PHB-5 & 87.06 & -0.60 & 3.57 & 0.76 \\
PHB-HV- N & 86.34 & -0.75 & 1.88 & 0 \\
PHB-HV-1 & 85.22 & -0.62 & 2.50 & 1.29 \\
PHB-HV-3 & 85.57 & -0.61 & 2.37 & 0.92 \\
PHB-HV-5 & 86.01 & -0.63 & 2.53 & 0.74 \\
\hline
\end{tabular}

Table 6 Static contact angles measured for PHB and PHBHV nanocomposites

\begin{tabular}{llll}
\hline Sample code & Left angle $(\theta)$ & Right angle $(\theta)$ & Mean value $(\theta)$ \\
\hline PHB-N & $76.21 \pm 1.10$ & $75.77 \pm 1.97$ & $75.99 \pm 0.31$ \\
PHB-1 & $82.11 \pm 1.37$ & $81.12 \pm 1.15$ & $81.62 \pm 0.70$ \\
PHB-2 & $79.70 \pm 1.90$ & $80.90 \pm 2.97$ & $80.30 \pm 2.26$ \\
PHB-3 & $73.21 \pm 0.84$ & $75.16 \pm 0.38$ & $74.19 \pm 1.38$ \\
PHB-5 & $74.14 \pm 2.21$ & $73.21 \pm 2.31$ & $73.68 \pm 0.66$ \\
PHBHV-N & $83.34 \pm 0.99$ & $82.93 \pm 1.85$ & $83.13 \pm 0.28$ \\
PHBHV-1 & $87.43 \pm 1.25$ & $85.46 \pm 1.03$ & $86.44 \pm 1.40$ \\
PHBHV-3 & $82.91 \pm 1.79$ & $82.00 \pm 2.85$ & $82.45 \pm 0.64$ \\
PHBHV-5 & $75.20 \pm 0.73$ & $74.65 \pm 0.26$ & $74.93 \pm 0.39$ \\
\hline
\end{tabular}

\section{Color Measurement}

Color changes in PHB and PHBHV nanocomposites due to addition of fillers are also very important for their possible use as food and medical packaging film since the commercial plastics (e.g. PP) used in packaging applications are generally transparent. In this manner, the effect of Cloisite 10A loading on color properties of pristine PHB and PHBHV composite films were evaluated by using Hunter Method. The total color differences $(\Delta E)$ of the coated films were calculated using the parameters $\mathrm{L}$, a, and $\mathrm{b}$. The evaluated color differences in nanocomposites were tabulated in Table 5 . The lightness parameter $\mathrm{L}$ did not change significantly by addition of clay into PHB and PHBHV matrix. The parameters " $a$ and b", which are the measure of redness-greenness, yellowness, respectively, also did not change significantly compared to pristine PHB and PHBHV samples. When total color difference is greater than three $(\Delta \mathrm{E}>3)$, the changes in the color can be recognized by human naked eye and below this limit it is acceptable in many applications [35].

Since the total difference values were maximum 1.29 , it can be said that the color change is insignificant due to the fine delamination of nanoclay platelets within the polymer matrix as it was also evidenced from the XRD and STEM results.

\section{Contact Angle Measurements}

Measured water contact angles of PHB and PHBHV nanocomposites were tabulated in Table 6 . The water contact angle of pristine PHB-N sample was $75.99^{\circ}$. However, when the small amount of Cloisite 10A (1 and 2\% w/w) was incorporated, the water contact angles of PHB nanocomposites significantly increased, and it reached its highest value of $81.62^{\circ}$, in comparison to pure PHB sample. This implies decrease in degree of wetting for PHB-1 and PHB-2 samples due to the exfoliation of layered silicates so as the surface became more hydrophobic. In addition, at higher amount of Cloisite 10A loading $(7 \% \mathrm{w} / \mathrm{w})$, the water contact angle decreased to $73.6^{\circ}$ and became even lower than pristine PHB samples. Similar trend was also observed for the PHBHV nanocomposites. Unmodified clay particles exhibited hydrophilic characteristic due to hydroxyl groups and addition to polymer matrix resulted in lower contact angle measurements [36]. Due to non-polar characteristic of organic modifier, incorporation of OMMT into polymer matrix increased the surface hydrophobicity. Diverse effect of OMMT at higher concentrations ( $5 \mathrm{w} / \mathrm{w} \%$ ) could be due to dispersion inefficiency resulted in poor interaction of polymer chains and organic modifier. The trend in contact angle measurements was in good accordance with the water vapour permeability measurements with supporting the idea of effect of dispersion level on surface hydrophobicity.

\section{Conclusion}

In this study, PHB and PHBHV based bionanocomposite films were prepared by solvent casting method with addition of Cloisite 10A at different weight percentages for potential packaging films. The study was focused on the investigation of effects of clay concentration and its dispersion as well as temperature on the water vapour barrier properties of the prepared nanocomposite films. Moreover, comprehensive characterization including thermal, mechanical, optical and structural properties of the nanocomposite films were performed. In this manner, this article provides a conduction between the exhibited properties and structure of nanocomposites of PHB and PHBHV. From the results, it was found that properties of PHB and PHBHV nanocomposite films were highly depended on the level of dispersion of Cloisite 10A in polymer matrix. As a result of XRD analysis, low content of clay loaded samples exhibited exfoliated structure of layered silicates thereby the properties of prepared nanocomposite films 
shows enhanced properties. Addition of just $1 \mathrm{wt} \%$ of clay particles enhanced the water vapor barrier properties of native PHB and PHBHV films by decreasing up to 41 and $25 \%$, respectively. The decrease in permeability of water is attributed to increment, in the tortuosity of permeable channel formed due to the clay delamination. Therefore, it was found that the level of intercalation/exfoliation has important effect on the barrier property improvements.

Cussler model showed that tortuosity increased by the random or regular dispersion of layered silicates with the aspect ratio of 160 which is good agreement with the reported aspect ratio range (10-1000) for layered silicates in the literature. Regarding to the all mechanical properties obtained, the maximum improvement was achieved for $3 \mathrm{wt} \%$ clay loaded samples for both PHB and PHBHV polymer composites. An increase of about 152.3 and $73.2 \%$ in tensile strength and of strain at break 77.4 and $17.5 \%$ was achieved for PHB and PHBHV polymers, respectively. The enhancement in mechanical properties were also attributed to dispersion level of layered silicates for, at higher amount of clay loaded samples both mechanical and barrier performance were diminished. Moreover, the enhancement of thermal properties were observed with the restriction of the mobility of the polymer chains due to exfoliated structure of layered silicates.

In summary, the prepared eco-friendly bio-based nanocomposites exhibited enhanced water vapor barrier, mechanical and optical properties, thereby showing potential for use as barrier films in food or medical packaging applications.

Acknowledgements The National Research Council of Turkey (Tübitak) is gratefully acknowledged for supporting this Project. (\#108M335). The authors are grateful to Professor Orhan Oztürk in the Physics Department of İzmir Institute of Technology (Iztech) for XRD characterization of the samples and to Material Research Center of İztech for STEM analysis.

\section{References}

1. Haugaard VKU, Mortensen AM, Hoegh G, Petersen L, Monahan KF (2001) In: Biobased packaging materials for the food industry-status and perspectives. KVL Department of Dairy and Food Science, Copenhagen

2. Platt DK (2006) In: Biodegradable polymers market report. Smithers Rapra Limited, Shropshire
3. Sanchez-Garcia MD, Gimenez E, Lagaron JM (2008) J Appl Polym Sci 108:2787

4. Mai Y-W, Yu Z-Z (2006) Polymer nanocomposites. Woodhead Publishing Limited, Cambridge

5. Zaikov GE, Pankova YN, Shchegolikhin AN, Iordanskii AL, Zhulkina AL, Ol'khov AA (2010) J Mol Liq 156:65

6. Miguel O, Egiburu JL, Iruin JJ (2001) Polymer 42:953

7. Botana A, Mollo M, Eisenberg P, Sanchez RMT (2010) Appl Clay Sci 47:263

8. Charlon S, Follain N, Chappey C, Dargent E, Soulestin J, Sclavons M, Marais S (2015) J Membr Sci 496:185

9. Botana A, Mollo M, Eisenberg P, Torres Sanchez RM (2010) Appl Clay Sci 47:263

10. Picard E, Vermogen A, Gerard JF, Espuche E (2007) J Membr Sci 292:133

11. Erceg M, Kovacic T, Klaric I (2009) Thermochim Acta 485:26

12. Cretois R, Follain N, Dargent E, Soulestin J, Bourbigot S, Marais S, Lebrun L (2014) J Membr Sci 467:56

13. Mittal V (2010) Optimization of polymer nanocomposite properties. Wiley, Weinheim

14. Wang S, Song C, Chen G, Guo T, Liu J, Zhang B, Takeuchi S (2005) Polym Degrad Stab 87:69

15. Erceg M, Kovacic T, Perinovic S (2010) Polym Compos 31:272

16. Oguzlu H, Tihminlioglu F (2010) Macromol Symp 298:91

17. Bordes P, Pollet E, Bourbigot S, Avérous L (2008) Macromol Chem Phys 209:1473

18. D’Amico DA, Manfredi LB, Cyras VP (2012) J Appl Polym Sci 123:200

19. Carli LN, Crespo JS, Mauler RS (2011) Compos Part A 42:1601

20. Choi WM, Kim TW, Park OO, Chang YK, Lee JW (2003) J Appl Polym Sci 90:525

21. Koo JH, (2006) Polymer nanocompomposites processing, characterization, applications, CRC Press, Boca Raton

22. Cervantes-Uc JM, Cauich-Rodriguez JV, Vazquez-Torres H, Garfias-Mesias LF, Paul DR (2007) Thermochim Acta 457:92

23. Gatos KG, Karger-Kocsis J (2007) Eur Polym J 43:1097

24. Paul DR, Robeson LM (2008) Polymer 49:3187

25. Luecha J, Sozer N, Kokini JL (2010) J Mater Sci 45:3529

26. Mittal V (2010) Barrier resistance generation in polymer nanocomposites. Wiley, New Jersey

27. Sun LY, Boo WJ, Clearfield A, Sue HJ, Pham HQ (2008) J Membrane Sci 318:129

28. Nielsen LE (1967) J Macromol Sci A 1:929

29. Eitzman DM, Melkote RR, Cussler EL (1996) Aiche J 42:2

30. Lape NK, Nuxoll EE, Cussler EL (2004) J Membrane Sci 236:29

31. Bharadwaj RK (2001) Macromolecules 34:9189

32. Herrera-Alonso JM, Marand E, Little J, Cox SS (2009) Polymer 50:5744

33. Hulsmann P, Philipp D, Kohl M (2009) Rev Sci Instrum 80:113901

34. Ikejima T, Yagi K, Inoue Y (1999) Macromol Chem Phys 200:413

35. Hong SI, Lee JW, Son SM (2005) Packag Technol Sci 18:1

36. Puglia D, Fortunati E, D’Amico DA, Manfredi LB, Cyras VP, Kenny JM (2014) Polym Degrad Stab 99:127 